\title{
Estudio de la Pureza Óptica de Citronelal presente en los Aceites Esenciales obtenidos de Citronela y de Eucalipto Citriodora
}

\author{
Silvia N. Zambón ${ }^{(1)}$, Ester R. Chamorro(1) y Sandra C. Casuscelli(2) \\ (1) QUIMOBI, Univ. Tecnológica Nacional, Facultad Regional Resistencia, French, 414, 3500, Resistencia- \\ Argentina. (e-mail: silzambon@frre.utn.edu.ar; snzambon@gmail.com; mchamorro@frre.utn.edu.ar) \\ (2) CITEQ, Univ. Tecnológica Nacional, Facultad Regional Córdoba, Maestro López y Cruz Roja Argentina, \\ 5016, Córdoba, Argentina. (e-mail: scasuscelli@scdt.frc.utn.edu.ar)
}

Recibido Ene. 5, 2015; Aceptado Mar. 5, 2015; Versión final Abr. 7, 2015, Publicado Ago. 2015

\begin{abstract}
Resumen
El objetivo del trabajo fue conocer la pureza óptica del citronelal presente en los aceites esenciales de citronela (Cymbopogon nardus (L.) Rendle) y eucalipto citriodora (Eucalyptus citriodora Hook), midiendo la rotación óptica específica. Los aceites esenciales se extrajeron por destilación por arrastre con vapor de agua a partir de variedades botánicas autenticadas. El citronelal, de ambos aceites esenciales fue separado por destilación fraccionada al vacío y el de Eucalyptus citriodora debió luego purificarse químicamente antes de realizar la medición de la rotación óptica específica. La identificación de los componentes de los aceites esenciales y la cuantificación del citronelal se realizaron por cromatografía gaseosa. La rotación óptica específica se determinó utilizando la Norma IRAM-SAIPA №18507-2002. En este estudio se obtuvo aproximadamente $95 \%$ de (+)-citronelal a partir de citronela, mientras que el citronelal del eucalipto citriodora es prácticamente racémico, lo cual está acuerdo con información de la literatura.
\end{abstract}

Palabras clave: citronelal, mezcla racémica, pureza óptica, Cymbopogon nardus (L.) Rendle, Eucalyptus citriodora Hook

\section{Optical Purity Study of Citronellal present in the Essential Oils obtained from Citronella and of Lemon Eucalyptus}

\begin{abstract}
The objective of the study was to determine the optical purity of citronellal present in the essential oils obtained from citronella (Cymbopogon nardus (L.) Rendle) and eucalyptus citriodora (Eucalyptus citriodora Hook), measuring the specific optical rotation. The essential oils are extracted by distillation by stripping with steam from authenticated botanical varieties. Citronellal from both essential oil was separated by fractional distillation under vacuum and the lemon eucalyptus product had to be chemically purified before measuring its specific optical rotation. The identification of the components of essential oils and the quantification of citronellal were performed by gas chromatography. The specific optical rotation was determined using the IRAM-SAIPA standard №18507-2002. In this study the citronellal obtained from citronella was about $95 \%$ of (+)-citronellal, while the citronellal obtained from eucalyptus citriodora was practically racemic, in agreement with information from the literature.
\end{abstract}

Keywords: citronellal, racemic mixture, optical purity, Cymbopogon nardus (L.) Rendle, Eucalyptus citriodora Hook 


\section{INTRODUCCIÓN}

Los aceites esenciales son productos del metabolismo secundario de las plantas, compuestos generalmente por terpenos, que están asociados o no a otros componentes. La mayoría de los aceites esenciales son volátiles, y responsables de las características sensoriales de dicho vegetal, tal como el aroma o el sabor (Bandoni, 2003), (Guenther, 1952). Estos compuestos volátiles son empleados en una amplia gama de industrias; entre las que se encuentran la farmacéutica, la de los aromas y sabores, de cuidado personal, de productos para el hogar, entre otras (Bandoni, 2003).

Se denomina citronela a varias especies de Cymbopogon entre los cuales se encuentra el Cymbopogon nardus (L.) Rendle; sin embargo, de la familia de las Myrtaceae está el eucalipto citriodora (Eucalyptus citriodora Hook), ambas, son especies aromáticas que se cultivan en regiones tropicales y subtropicales del planeta y tienen bajo precio en el mercado internacional (Bizzo y col, 2009). Estudios realizados indican que los aceites esenciales de citronela y eucalipto citriodora poseen actividad biológica. Además, tienen la propiedad de ser repelentes naturales de insectos (Kim y col, 2005), (Makhaik y col, 2005), por lo que cobra especial relevancia en las regiones de clima tropical y subtropical donde se sufre de enfermedades endémicas como fiebre amarilla, dengue, malaria y otras generadas por mosquitos vectores (Gutiérrez y col, 2008). Martins estudió la acción acaricida del aceite esencial de citronela contra la garrapata Boophilus microplus (Martins, 2006); Sanchez-Garcia y col. analizaron la acción antimicrobiana del mismo aceite esencial sobre contaminantes del cultivo in vitro de plantas (Sanchez-García y col, 2007). Singh trabajó sobre la actividad antioxidante del aceite esencial de eucalipto citriodora y sus componentes (Singh y col, 2012) y Luqman sobre su acción antimicrobiana, contra algunas especies de levaduras, hongos y bacterias (Luqman, 2008).

Los aceites esenciales de citronela y eucalipto citriodora tienen en común, un componente principal, el citronelal. La cantidad de este componente en los aceites esenciales varía con la especie, así la primera contiene entre 30 a 45\% (Cassel, 2006); (Guenther, 1952); (Bandoni, 2003); (Berger, 2007) y la segunda entre 75 a $85 \%$ (Keszei y col, 2010). El citronelal (3,7-dimetil-6-octenal) es un monoterpeno lineal que posee dos isómeros ópticos, (-)-citronelal y (+)-citronelal, es uno de los componentes responsables de la actividad biológica de las especies mencionadas (Can Bas y col, 2010); (Kim y col, 2005). La Figura 1 muestra la conformación más estable del (+)-citronelal, calculada en el vacío empleando la Teoría del Funcional de la densidad con el funcional híbrido triparamétrico de Becke y el funcional de correlación de Lee, Yang y Parr conocido como (B3LYP), se utilizó el conjunto base $6-31+G(d, p)$, empleando el programa Gaussian03. Es importante señalar que el citronelal proveniente de la citronela es casi exclusivamente dextrógiro, mientras que el del eucalipto citriodora es una mezcla cuasiracémica (Guenther, 1952).

La industria de aromas y fragancias tiene particular interés en el desarrollo de productos sintéticos a partir de materiales de bajo costo y con alta pureza óptica. La síntesis química convencional, tiende a dar lugar a mezclas racémicas y esto puede ser extremadamente perjudicial para el aroma o la actividad biológica de un producto de interés. También puede ser bastante engorrosa y costosa la separación de los compuestos de la mezcla resultante. Existen varios trabajos que estudian la identificación y cuantificación de los isómeros de citronelal (Cahyono y col, 2013); (Bonaccorsi y col, 2011). Dado que la cromatografía de gases convencional no resulta adecuada para la separación de los isómeros, Bonaccorsi y col utilizan la cromatografía de gases multidimensional (MDGC) (Bonaccorsi y col, 2011), (Cahyono y col, 2013). Aunque este es un método complejo y de elevado costo, y considerando que ambos aceites esenciales tienen bajo precio en el mercado internacional (Can Bas y col, 2010), seguramente se justifica plenamente el gasto de la aplicación de esta metodología, cuando sea necesario realizar muchos análisis de este tipo o para otros aceites de mayor precio de mercado.

La rotación óptica específica es una propiedad tan importante y característica de un compuesto como lo son sus puntos de fusión, de ebullición y su densidad. Así, el conocimiento de la pureza óptica específica, definida como la relación entre la rotación óptica específica medida y la del enantiómero puro, permite determinar distintos tipos de adulteraciones, tales como adición de compuestos sintéticos o componentes de diferentes orígenes botánicos, los cuales podrían dañar drásticamente las propiedades biológicas u olfatorias, íntimamente relacionadas con la naturaleza estereoquímica de los mismos. Por ejemplo, el valor de la rotación óptica específica del (+)-citronelal es $+12,5^{\circ}$ (Bedoukian, 1986), mientras que para mezclas racémicas es, lógicamente $0^{\circ}$. La rotación óptica específica del citronelal asociada a una conveniente purificación, permite la cuantificación de la pureza óptica del mismo. En el presente trabajo se evaluó la pureza óptica del citronelal presente en los aceites esenciales de citronela (Cymbopogon nardus (L.) Rendle) y eucalipto citriodora (Eucalyptus citriodora, Hook), del noreste argentino, determinando la rotación óptica específica de los componentes aislados. 


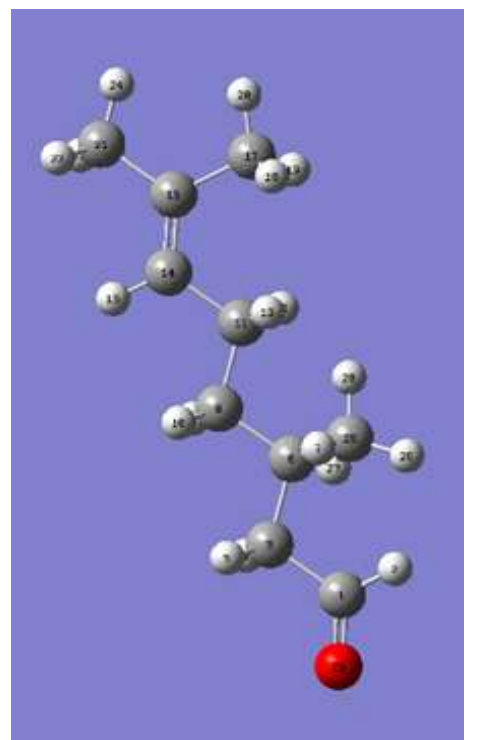

Fig. 1. Conformación más estable del (+)-citronelal

\section{MATERIALES Y MÉTODOS}

Se presentan detalles sobre el material vegetal estudiado, la destilación por arrastre con vapor, la destilación fraccionada al vacío, la purificación del citronelal, el análisis cromatográfico y la determinación de la pureza óptica.

\section{Material Vegetal}

Las especies estudiadas fueron Cymbopogon nardus (L.) Rendle proveniente de Colonia Benítez en la provincia del Chaco y Eucalyptus citriodora Hook, de Paso de la Patria en la provincia de Corrientes, ambas localidades de la región Noreste de la República Argentina. Las especies vegetales fueron autenticadas por el Instituto de Botánica del Noreste (IBONE) que pertenece al CONICET en la Universidad Nacional del Noreste en la ciudad de Corrientes, Argentina y las muestras de referencia fueron almacenadas en su Herbario, bajo los números de referencia CTES0043467 para el Cymbopogon nardus (L.) Rendle y CTES0045208 para el Eucalyptus citriodora Hook. Se recolectó el material vegetal en el mes de marzo alrededor de las 10 de la mañana, de cinco parcelas de la plantación y se realizaron dos destilaciones por parcela y por especie. Se cosecharon las hojas en el caso de la citronela y del eucalipto citriodora además de las hojas, las ramas finas de hasta aproximadamente $10 \mathrm{~mm}$ de espesor, en muy baja proporción. Seguidamente se pesó y se dejó orear en un lugar oscuro y seco, hasta alcanzar aproximadamente el $40 \%$ de su peso fresco.

\section{Destilación por arrastre con vapor}

El equipo utilizado para la destilación por arrastre con vapor de agua constó de un tanque destilador, donde se colocó $5 \mathrm{~kg}$ del material vegetal oreado. El mismo se conectó a una caldera humutubular de baja presión y al intercambiador de calor de casco y tubos. La separación de las fases se realizó por medio de un frasco florentino adaptado, los aceites esenciales se filtraron y se secaron con sulfato de sodio anhidro, luego se los almacenó en recipientes de color caramelo y en ambiente refrigerado para su buena conservación.

Todas las destilaciones fueron analizadas por cromatografía de gases acoplada a un detector de ionización de llamas, las repeticiones fueron agrupadas en el mismo frasco color caramelo, porque presentaban perfiles cromatográficos similares. Además, muestras de ambos aceites esenciales fueron analizadas por cromatografía en fase gaseosa, acoplada a un espectrómetro de masas para poder identificar los componentes.

\section{Destilación fraccionada al vacío}

Se pesó $100 \mathrm{~g}$ del aceite esencial obtenido de las dos destilaciones de cada parcela en un al balón de alimentación de $300 \mathrm{ml}$. Repitiéndose el mismo procedimiento para las parcelas restantes, por cada especie. La separación del citronelal se realizó por destilación fraccionada al vacío, en una columna empacada de aproximadamente 9 platos teóricos. El empaque es un relleno similar a los de montura de acero inoxidable, donde la primera fracción sale alrededor de los 38 a $40^{\circ} \mathrm{C}$, la segunda alrededor de 58 a $60^{\circ} \mathrm{C}$ y la tercera alrededor de $90^{\circ} \mathrm{C}$, como los componentes principales de ambas especies son similares, destilan a aproximadamente las mismas temperaturas. La presión de trabajo fue de $4 \mathrm{~mm} \mathrm{Hg}$. 
Se tomó la segunda fracción de 5 rectificaciones de aceite de Cymbopogon nardus. Se obtuvieron de esta forma 5 muestras de citronelal enriquecido de Cymbopogon nardus. Estas fueron analizadas por cromatografía de gases con un detector de ionización de llama, se midió la rotación óptica y la densidad con lo cual se calculó la rotación óptica específica y la pureza óptica. Para el Eucalyptus citriodora Hook fueron utilizadas las mismas condiciones de proceso que para Cymbopogon nardus. Sin embargo, en este caso, fue necesario la purificación del citronelal de la segunda fracción, obtenida de la rectificación del aceite esencial antes de llevar a cabo la determinación de la rotación óptica.

\section{Purificación del citronelal de Eucalyptus citriodora}

La segunda fracción obtenida de la rectificación del aceite esencial de Eucalyptus citriodora fue purificada utilizando la metodología definida por Bedoukian (Bedoukian, 1986) y por Guenther (Guenther, 1952), pero introduciendo algunas modificaciones que se detallan a continuación. Se utilizó solución al $40 \% \mathrm{P} / \mathrm{V}$ de bisulfito de sodio recientemente preparada e hidróxido de sodio $1 \mathrm{~N}$. A un erlenmeyer de $250 \mathrm{ml}$ se agregó $20 \mathrm{~g}$ de la segunda fracción obtenida de la rectificación del aceite esencial, $50 \mathrm{ml}$ de solución de bisulfito de sodio y $5 \mathrm{ml}$ de solución de hidróxido de sodio $1 \mathrm{~N}$. Se agitó y se dejó reaccionar hasta la formación de un precipitado blanco. Una vez culminada la reacción se colocó $30 \mathrm{ml}$ de hexano y $10 \mathrm{ml}$ de agua destilada para disolver en parte la sal formada. El precipitado, que contenía el citronelal al que se adiciona el ion bisulfito, se separó por filtración y lavó 10 veces con $5 \mathrm{ml}$ de hexano. El líquido separado, que contenía una mezcla de alcoholes, en su mayoría isopulegol y citronelol, ya que todos los compuestos carbonílicos quedan en la fase sólida, fue analizado por cromatografía de gases acoplada a un detector de ionización de llamas.

El citronelal fue regenerado, tratando el precipitado con $30 \mathrm{ml}$ de agua destilada, $30 \mathrm{ml}$ de hexano y $30 \mathrm{ml}$ de hidróxido de sodio $1 \mathrm{~N}$, a $40^{\circ} \mathrm{C}$ con agitación, durante una hora, momento en el que se agregaron nuevamente $20 \mathrm{ml}$ de hexano y $20 \mathrm{ml}$ de hidróxido de sodio $1 \mathrm{~N}$ y por último $20 \mathrm{ml}$ más de hexano. Cuando todo el precipitado se disolvió, se separaron las fases y se recuperó el citronelal de la fase orgánica evaporando el solvente. De esta forma de obtuvieron las 5 muestras de citronelal purificado del Eucalyptus citriodora, las que fueron analizadas por cromatografía de gases acoplada a un detector de ionización de llama, y se midió la rotación óptica y la densidad, con lo cual se calculó la rotación óptica específica y la pureza óptica.

\section{Análisis cromatográfico}

La identificación de los componentes se realizó utilizando un cromatógrafo Agilent 7890 que contó de una columna de metilsilicona, $30 \mathrm{~m} \times 0,25 \mathrm{~mm}$, i.d. $0,2 \mu \mathrm{m}$, con el siguiente programa de temperatura,70ㄷ por $5 \mathrm{~min}$, con una rampa de $5^{\circ} \mathrm{C} / \mathrm{min}$ hasta $280^{\circ} \mathrm{C}$, acoplado con un detector selectivo de masas Agilent 5975 . Las condiciones de operación para este detector fueron ionización electron con un EM Voltage Scanmode: 1923 V, con un barrido de 50-550 amu y un muestreador automático, las bibliotecas utilizadas fueron F.W. McLafferty, D.B. Stauffer, Wiley/NBS Registry of MassSpectral Data, Wiley, New York, 1989.

Para la cuantificación del contenido de citronelal de las muestras se utilizó metil etil cetona como estándar interno en un cromatógrafo de gases SHIMADZU GC 14B utilizando una columna Megabore DB-WAX P / N 125-7032 conectada a un detector de ionización de llama. La temperatura del detector fue $220^{\circ} \mathrm{C}$ y la del inyector fue $180^{\circ} \mathrm{C}$. El programa de temperatura utilizado fue $30^{\circ} \mathrm{C}$ mantenido durante $2 \mathrm{~min}$, la rampa de $5^{\circ} \mathrm{C} / \mathrm{min}$ hasta alcanzar los $200^{\circ} \mathrm{C}$, donde al final se mantuvo durante $5 \mathrm{~min}$. Las muestras fueron medidas por duplicado.

\section{Determinación de la pureza óptica}

Para la determinación de la rotación óptica específica se utilizó la metodología definida en la Norma IRAMSAIPA №18507-2002con un polarímetro de disco tipo WXG-4 marca Hedao de 0,001 de precisión. La densidad relativa a $20^{\circ} \mathrm{C}$ se obtuvo mediante la Norma IRAM-SAIPA N ${ }^{\circ} 18504-2002$ utilizando una balanza analítica Denver instrument de $0,1 \mathrm{mg}$ de precisión. La rotación óptica específica $\alpha_{\mathrm{D}}^{20}$ a $20^{\circ} \mathrm{C}$ fue calculada utilizando la densidad relativa a $20^{\circ} \mathrm{C}$ y el tubo del polarímetro de $1 \mathrm{dm}$. Se utilizó la siguiente ecuación:

$\left[\alpha_{\mathrm{D}}^{20}\right] \mathrm{obs}=\frac{\alpha}{\mathrm{L} . \delta}$

Donde a es la rotación óptica medida en el polarímetro $\left[^{\circ}\right], \mathrm{L}$ es el largo del tubo [dm] y $\delta$ es la densidad relativa en $[\mathrm{g} / \mathrm{ml}]$. Se utilizó como valor de referencia de la rotación óptica específica del $(+)$-citronelal ( $\left[\alpha_{\mathrm{D}}^{20}\right]$ ref $)$ al valor medio de $+12.5^{\circ}$ definido por Bedoukian (Bekoukian, 1986). 
La pureza óptica (p.o.) fue determinada con las fórmulas:

$$
\begin{aligned}
& \text { p.o. }=\left[\mathrm{\alpha}_{\mathrm{D}}^{20}\right] \mathrm{obs} /\left[\mathrm{\alpha}_{\mathrm{D}}^{20}\right] \mathrm{ref} \times 100 \\
& \text { p.o. }=\frac{d-l}{d+l} \times 100
\end{aligned}
$$

Donde $d$ y $l$ corresponden a la cantidad de los isómeros puros expresada como masa, porcentajes o concentraciones.

\section{RESULTADOS Y DISCUSIÓN}

\section{Caracterización química de los aceites esenciales}

La identificación de los componentes se realizó por medio de un cromatógrafo de gases acoplado a un detector selectivo de masas. Los diferentes compuestos con sus tiempos de residencia y las cantidades relativas de los mismos se observan en la Tabla 1. En el caso del aceite esencial de Cymbopogon nardus (L.) Rendle el componente mayoritario es el citronelal, seguido por el geraniol y el citronelol. Estos valores están de acuerdo a los valores determinados por Berger para la especie de Java, Cymbopogon winterianus, citronelal (30-45\%), geraniol (20-25\%), citronelol (9-15\%) pero en desacuerdo con los valores determinados para Cymbopogon nardus (L) W.Wats, de Ceylon, citronelal (3-6\%), geraniol 15-23\%), citronelol (3-9\%) (Berger, 2007). Cassel y col., estudian la variedad Cymbopogon winterianus del sur de Brasil, aunque está de acuerdo con que el componente mayoritario es el citronelal, aunque en menores proporciones (35,9\%), mientras que el citronelol 5,2\%, geraniol $20,9 \%$, y otros componentes de interés como el acetato de geranilo $4 \%$, a-cardinol 8\%, germacreno B 6,8\%, $\alpha$-cardinol 8\% (Cassel y col., 2006). Wei y col., caracterizaron el aceite esencial de Cymbopogon nardus de Malasia también obtuvieron como componente mayoritario al citronelal 29,6\%, sin embargo no está de acuerdo a que los siguientes componentes mayoritarios sean citronelol y geraniol ya que para este caso son citral 11\% y isobutirato de 3,7-dimetil-2,6-octadienilo 6,9\%. (We y col., 2012) Es importante resaltar que la Norma IRAM-SAIPA №18529 $3^{\mathrm{a}}$ Ed. (2006) especifica para el aceite esencial de Cymbopogon winterianus de Argentina, los valores de citronelal (35-45\%), geraniol (19$24 \%)$, citronelol (9-14\%), limoneno (1,5-5\%), linalool (0,2-2\%), isopulegol $(0,2-1 \%)$, elemol $(2-4 \%)$, estando los valores obtenidos en este estudio, dentro de esta especificación.

Por otra parte para el aceite esencial de Eucalyptus citriodora Hook el componente mayoritario por excelencia es el citronelal seguido por el citronelol y los isómeros de isopulegol, estos resultados coinciden con los valores obtenidos por Singh y col., que analizan este aceite esencial proveniente de la India, con una diferencia importante en el contenido de citronelal, ya que sigue siendo el componente mayoritario pero informan $60,66 \%$ de citronelal; $8,19 \%$ de isopulegol; $0,58 \%$ de isoisopulegol; $12,58 \%$ de $\beta$-citronelol y $2,87 \%$ de p-metano-3,8-diol (Singh, 2012). Los valores obtenidos en el presente trabajo son diferentes a los obtenidos por El-Mageed y col., ya que el aceite esencial de Eucalyptus citriodora obtenido de Egipto posee como componente principal el 3-hexeno-1-ol con 31,26\%; cis-geraniol con 19,66\%, acetato de citronelol con 13,68\%, 5-hepten-1-ol 3-6 dimetilo con 13,14\% y citronelal con 9,36\% (El-Mageed y col., 2011). Sin embargo, Castro y col., estudiaron la influencia en el rendimiento y la composición química de las hojas del Eucalyptus citriodora Hook, obtenidas de diferentes épocas del año en municipios de Minas Gerais, observándose en agosto disminuye el contenido de citronelal, pero se mantiene como el componente mayoritario (Castro y col., 2008). Hay que remarcar que la citronela, no se puede cortar en invierno porque es sensible a las heladas. Es importante señalar que el Eucalyptus citriodora Hook, contiene un máximo de $10 \%$ de isopulegoles (NF ISO 3044-1997), significativamente diferente al Cymbopogon nardus que tiene un

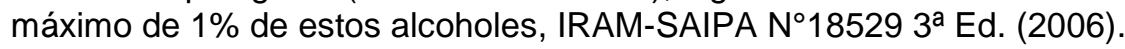

\section{Análisis de los resultados}

En la Tabla 2, se observa que los valores de concentración alcanzados de citronelal por enriquecimiento y purificación de los aceites esenciales de las especies Cymbopogon nardus (L.) Rendle y Eucalyptus citriodora Hook son de 96,7 a 98,5 \%P/P y 96,8 a 98,5\%P/P respectivamente. En cuanto a la rotación óptica específica los valores medidos para el Cymbopogon nardus (L.) Rendle, van desde $11,14^{\circ}$ a $11,51^{\circ}$, aumentando a medida que aumenta la concentración de citronelal en las muestras, con un valor promedio medido de $11,32^{\circ} \pm 0,13^{\circ}$ cercano al valor de referencia de $12,5^{\circ}$ (Bedoukian, 1986). En el caso del Eucalyptus citriodora Hook, los valores de la rotación óptica específica van desde $0,12^{\circ} a \quad 0,91^{\circ}$, disminuyendo a medida que aumenta el valor de la concentración de citronelal en las muestras, el valor promedio es de $0,45^{\circ} \pm 0,31^{\circ}$ muy cercano al valor de rotación óptica específica de mezclas racémicas. 
Tabla 1: Composición química de los aceites esenciales de Cymbopogon nardus y Eucalyptus citriodora

\begin{tabular}{|c|c|c|c|c|c|}
\hline \multicolumn{3}{|c|}{ Aceite esencial de citronela (C. nardus) } & \multicolumn{3}{|c|}{ Aceite esencial de eucalipto citriodora ( $E$. citriodora) } \\
\hline $\begin{array}{l}\text { Tiempo de } \\
\text { retención (min) }\end{array}$ & $\begin{array}{l}\text { Cantidades } \\
\text { relativas (\%) }\end{array}$ & Identificación & $\begin{array}{l}\text { Tiempo de } \\
\text { retención (min) }\end{array}$ & $\begin{array}{l}\text { Cantidades } \\
\text { relativas (\%) }\end{array}$ & Identificación \\
\hline 5,92 & 4,39 & limoneno & 5,9 & 0,28 & 1,8-cineol \\
\hline 8,08 & 49,6 & citronelal & 5,01 & 90,18 & citronelal \\
\hline 8,31 & 0,47 & isopulegol & 8,31 & 2,50 & isopulegol \\
\hline 9,72 & 12,32 & citronelol & 8,52 & 0,27 & neoisopulegol \\
\hline 10,25 & 17,52 & geraniol & 9,70 & 3,52 & citronelol \\
\hline 12,41 & 2,41 & beta elemeno & 10,1 & 2,04 & geraniol \\
\hline 12,96 & 1,71 & acetato de geranilo & 12,40 & 0,24 & beta elemeno \\
\hline 13,56 & 1,53 & d-cadimeno & 14,17 & 0,46 & cariofileno \\
\hline 16,51 & 3,41 & elemol & & & \\
\hline
\end{tabular}

Del mismo modo, la pureza óptica referida al (+)-citronelal del Cymbopogon nardus (L.) Rendle aumenta a medida que se incrementa la concentración del citronelal, alcanzando valores que van desde el 94,57\% hasta $96,02 \%$ con un valor promedio del $95,28 \% \pm 0,52$. Para el Eucalyptus citriodora Hook la pureza óptica varía entre $50,48 \%$ a $53,62 \%$ disminuyendo a medida que aumenta la concentración del citronelal. E valor promedio de la pureza óptica para esta última especie es de $51,80 \% \pm 1,26$. Comparando los valores de las purezas ópticas promedio de ambas especies se observa una marcada diferencia indicando que el aceite esencial de Cymbopogon nardus (L.) Rendle posee un alto grado de pureza óptica referida al (+)-citronelal mientras que el aceite esencial de Eucalyptus citriodora Hook presenta una mezcla cuasiracémica. Los resultados obtenidos coinciden con los obtenidos por Cahyono y col., ya que obtienen que el citronelal de la citronela posee $88,21 \%$ de exceso enantiomérico referidos al $(R)-(+)$-citronellal (Cahyono, 2013).

En ambas especies se observa la influencia de la pequeña cantidad de impurezas, tales como isopulegol, citronelol, geraniol, entre otros, sobre la actividad óptica; lo cual está de acuerdo a con la ley de Biot, quien define a la rotación óptica como inversamente proporcional a la concentración de la sustancia. El aumento de los valores de las desviaciones estándares, para el caso del Eucalyptus citriodora Hook demuestra que el proceso de purificación química, ausente en el caso del Cymbopogon nardus (L.) Rendle, genera mayor variabilidad en los resultados finales.

Tabla 2: Concentración del citronelal, rotación óptica específica y pureza óptica referida al isómero (+)citronelal de Cymbopogon nardus y Eucalyptus citriodora

\begin{tabular}{|c|c|c|c|c|c|c|}
\hline \multirow{2}{*}{ Muestra } & \multicolumn{2}{|c|}{ Citronelal enriquecido de la citronela } & \multicolumn{2}{c|}{ Citronelal purificado del eucalipto citriodora } \\
\cline { 2 - 7 } & $\begin{array}{c}\text { Concent. } \\
\text { citronelal } \\
(\% p / p)\end{array}$ & $\begin{array}{c}\text { Rot. óptica } \\
\text { específica }\end{array}$ & $\begin{array}{c}\text { Pureza óptica } \\
(+) \text {-citronelal (\%) }\end{array}$ & $\begin{array}{c}\text { Concentración } \\
\text { citronelal (\%P/P) }\end{array}$ & $\begin{array}{c}\text { Rot. óptica } \\
\text { específica }\end{array}$ & $\begin{array}{c}\text { Pureza óptica (+)- } \\
\text { citronelal } \\
(\%)\end{array}$ \\
\hline 1 & 96,7 & 11,14 & 94,57 & 96,8 & 0,91 & 53,62 \\
\hline 2 & 97,8 & 11,30 & 95,20 & 97,0 & 0,62 & 52,46 \\
\hline 3 & 98,1 & 11,31 & 95,25 & 97,8 & 0,36 & 51,45 \\
\hline 4 & 98,2 & 11,34 & 95,37 & 98,3 & 0,24 & 50,96 \\
\hline 5 & 98,5 & 11,51 & 96,02 & 98,5 & 0,12 & 50,48 \\
\hline Prom & 97,86 & 11,32 & 95,28 & 97,68 & 0,45 & 51,80 \\
\hline Desv. est. & 0,69 & 0,13 & 0,52 & 0,75 & 0,31 & 1,26 \\
\hline
\end{tabular}

\section{CONCLUSIONES}

Utilizando métodos simples de separación, combinados con el cálculo de la rotación óptica específica, se pudo determinar que el citronelal proveniente del Cymbopogon nardus (L.) Rendle tiene un alto contenido del isómero (+)-citronelal, y que el correspondiente al Eucalyptus citriodora Hook responde a una mezcla cuasiracémica. A pesar de que esta especie tiene menor proporción de citronelal, si se lo compara con el Eucalyptus citriodora Hook, la abundancia del isómero (+)-citronelal, le otorga mayor valor agregado cuando 
se lo destina a reactivo para síntesis química, ya que brinda mayor estereoespecíficidad hacia determinados productos de reacción. La determinación de la rotación óptica específica es una técnica válida para la evaluación de la calidad de los aceites esenciales estudiados.

\section{REFERENCIAS}

Bandoni, A., Los Recursos vegetales aromáticos en latinoamérica. Su aprovechamiento industrial para la producción de aromas y sabores, $2^{a}$ Edición. CYTED. Buenos Aires, Argentina (2003)

Bedoukian, P. Z., Perfumery and flavoring synthetics, 3a Edition. Allured Pub Corp. USA (1986)

Berger, R., Flavours and Fragrance Chemistry, Bioprocessing and Sustainability. Springer. Berlin, Alemania (2007)

Bizzo, H., Hovell, A., e Rezende, C., Óleos essenciais no Brasil: Aspectos gerais, desenvolvimento e perspectivas, Quimica Nova, 32 (3), 588-594 (2009)

Bonaccorsi, I., Sciarrone, D., Cotroneo, A., Mondello, L., Dugo, P., y Dugo, G..,Enantiomeric distribution of key volatile components in Citrus essential oils, Braz. J. Pharmacog., 841-849 (2011)

Cahyono, E., Pranowo, H., y Muchalal, T., Analysis of the enantiomers ratio of Citronellal from Indonesian Citronella Oil Using Enantioselective Gas Chromatography, Mal. J. Fund. Appl. Sci., 62-66 (2013)

Can Bas, K., and Buchbauer, G., Handbook of Essential Oils. Science, Technology and applications. Taylor and Francis Group, LLC. Florida, USA (2010)

Castro, N., Carvalho, G., Cardoso, M., Pimentel, F., Correa, R., Guimarães, L., Avaliação de rendimento e dos constituintes químicos do óleo essencial de folhas de Eucalyptus citriodora Hook. colhidas em diferentes épocas do ano em municípios de Minas Gerais, Rev. Bras. PI. Med., Botucatu, 10 (1), $70-75$ (2008)

Cassel, E. V., Experimental and modeling of the Cymbopogon winterianus. Essential oil extraction by steam distillation, J. Mex. Chem. Soc., 50 (3), 126-129 (2006)

El-Mageed, A., Osman, A., Tawfik, A., Mohammed, H., Chemical composition of the essential oils of four Eucalyptus Species (Myrtaceae) from Egypt. Res. J. Phytoch., 5 (2), 115-122 (2011)

Guenther, E., The essential oils, 3를 Edition. Volume four, Van Nostrand Company (1977)

Gutiérrez, M., y Herbosa, R., Enfermedades tropicales transmitidas por vectores. Medidas preventivas y profilaxis. Offarm, 27 (6), 78-87 (2008)

Keszei, A., Brubaker, C., Carter, R., Köllner, T., Degenhardt, J., and Foleya, W., Functional and evolutionary relationships between terpene synthases from Australian Myrtaceae. Phytochemistry (71), 844-852 (2010)

Kim, J., Kang, C., Lee, J., Kim, Y., Han, H., e Yun, H., Evaluation of Repellency Effect of Two Natural Aroma Mosquito Repellent Compounds, Citronella and Citronellal. Entomol. Res., 35 (2), 117-120 (2005)

Lenardao, E., Botteselle, G., Azambuja, F., Perin, G., y Jacob, R., Citronellal as key compound in organic synthesis. Tetrahedron (63), 6671-6712 (2007)

Luqman, S. D., Antimicrobial activity of Eucalyptus citriodora essential. IJEOT, 2, 69-75 (2008)

Makhaik, M., Narayan, S., y Tewary, D., Evaluation of anti-mosquito properties of essential oils. J. Sci. Ind. Res., 64, 129-133(2005)

Martins, R., Estudo in vitro da ação acaricida do óleo essencial da gramínea Citronela de Java (Cymbopogon winterianus Jowitt) no carrapato Boophilus microplus. Rev. Bras. PI. Med. , 26 (1), $71-78$ (2006)

Sanchez-García, C., y otros 6 autores, Evaluación del efecto del aceite esencial de Cymbopogon nardus para el control de microorganismos contaminantes del cultivo in vitro de plantas. Biotecnología vegetal, 7 (3), 187-190 (2007)

Singh, H., y otros 6 autores, Assessment of in vitro antioxidant activity of essential oil of Eucalyptus citriodora (lemon-scented Eucalypt; Myrtaceae) and its major constituents. Food sci. technol., 48, 237-241 (2012)

Wei, L., Wee, W., Chemical composition and antimicrobial activity of Cymbopogon nardus citronella essential oil against systemic bacteria of aquatic animals, Iran. J. Microbiol, 5 (2), 147-152 (2013) 
